\title{
Kinematics Analysis and Simulation of KUKA KR5-arc Welding Robot based on MATLAB
}

\author{
Sailong $\mathrm{Wu}^{1, \mathrm{a}}$, Qiang $\mathrm{Lin}^{2, \mathrm{~b}}$, Peng Wang ${ }^{3, \mathrm{c}}$ \\ ${ }^{1}$ Department of Mechanical \& Electrical and Information Engineering, China University of Mining \\ and Technology(Beijing), Beijing, 100083, China \\ ${ }^{2}$ Department of Mechanical \& Electrical and Information Engineering, China University of Mining \\ and Technology(Beijing), Beijing, 100083, China \\ ${ }^{3}$ Department of Mechanical \& Electrical and Information Engineering, China University of Mining \\ and Technology(Beijing), Beijing, 100083, China
}

aemail: wusailong1995@163.com, bemail:254887029@qq.com, 'email:1438225176@qq.com

Keywords: Welding Robot; Kinematics Analysis; KUKA; MATLAB

\begin{abstract}
Introduce the application situation and development trend of welding robot, selecting the KUKA KR5-arc as the research object, simplifying its mechanical system and using the Denavit-Hartenberg (D-H matrix) representation and homogenous matrix to build the kinematic transformation of 6-DOF articulated robots. With the help of the Robotic Toolbox of MATLAB, the trajectory of robot manipulator can be obtained by the simulation and the results will provide a basis for robot motion analysis and design.
\end{abstract}

\section{Introduction}

Robot is the embodiment of flexible automation technology [1] and is one of the most popular research directions of mechanical and electrical integration field. From the beginning, robots do not have any external information feedback ability and later robots are equipped with certain perception of the outside environment [2]. Now the mainstream products in the market are mostly the intelligent robots.

The welding robots account for about half of the total number of industrial robots [3], showing great advantages in replacing manual labor and reducing intensity of the workers, to ensure the quality of this craft and improve the efficiency of welding. Not only do robots need completing the welding tasks at a high speed, but also they are supposed to make the process smoothly and accurately, tracking every pose of the manipulator.

This paper aims to deal with the issues above, modeling KUKA KR5-arc welding robot based on $\mathrm{D}-\mathrm{H}$ coordinate system theory and analyzing the kinematics with the Robotic Toolbox in the MATLAB environment. The simulation obtained by MATLAB keeps track of the trajectory of the robot, recording the displacement and other kinematic parameters.

\section{KUKA KR5-arc in Details}

KUKA is one of the world's leading industrial robots companies and its main customers are from the automotive manufacturing field.

KR5-arc robot is particular, compact and lightweight, 6DOF. It is especially suitable for welding work, accomplishing the task in different uses reliably. The three-dimensional structure diagram of 6DOF and mechanical size are shown in Figure 1 (a) and (b). 


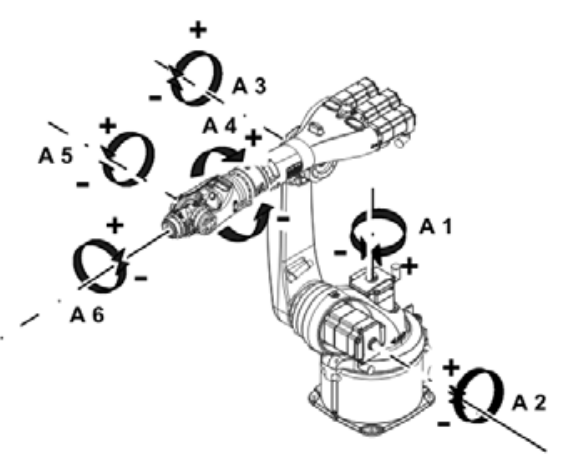

(a) 6DOF

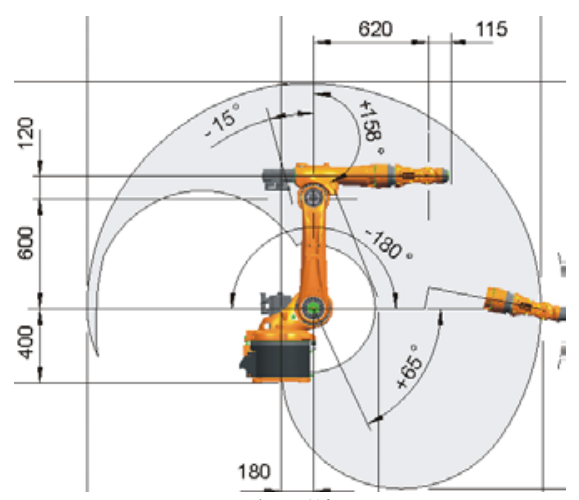

(b) Size

Fig.1. The mechanical data

\section{D-H matrix in Robot}

A robot manipulator consists of several links connected by usually single degree of freedom joint say, a revolute or a prismatic [4]. In order to control the end-effector with respect to the base and find out the robot's attitude, it is necessary to find the relation between the coordinate frames attached to the end-effector and the base.

In 1955 Denvit and Hartenberg put forward (D-H) matrix method-describing the coordinate frames attached transformations between the coordinate frames attached to all the links and forming the overall description in a recursive manner. For this purpose, the material presented in the previous section for describing the position and orientation of the rigid body is useful for obtaining the composition of coordinate transformations between the consecutive frames. Attached to the body coordinate system relative to the base are rotating and moving $4 \mathrm{x} 4$ homogeneous transformation matrix [5].

$$
\begin{aligned}
& \mathrm{T}_{5}^{0}=A_{1}^{0} A_{2}^{1} A_{3}^{2} A_{4}^{3} A_{5}^{4} \\
& T_{5}^{0}=\left[\begin{array}{cccc}
n_{x} & o_{x} & a_{x} & p_{x} \\
n_{y} & o_{y} & a_{y} & p_{y} \\
n_{z} & o_{z} & a_{z} & p_{z} \\
0 & 0 & 0 & 1
\end{array}\right] \quad A_{i}^{i-1}=\left[\begin{array}{cccl}
\cos \theta_{i} & -\sin \theta_{i} & 0 & l_{i} \cos \theta_{i} \\
\sin \theta_{i} & \cos \theta_{i} & 0 & l_{i} \cos \theta_{i} \\
0 & 0 & 1 & 0 \\
0 & 0 & 0 & 1
\end{array}\right]
\end{aligned}
$$

In the type(1): $T_{5}^{0}$ is the robot manipulator coordinate system relative to the position and orientation of the fixed coordinate system; $A_{1}^{0}$ is the body coordinate system relative to the base coordinate system; $A_{2}^{1}$ is the shoulder coordinate system relative to the trunk coordinate system; $A_{3}^{2}$ is the arm coordinate system relative to the shoulder system; $A_{4}^{3}$ is the wrist coordinate system relative to the arm coordinate system; $A_{5}^{4}$ is the robot manipulator system relative to the wrist coordinate system.

D-H matrix can be decomposed into the rotation matrix and the translation matrix relative to the fixed coordinate system. The two matrix determine the position and orientation of the robot manipulator. $\mathrm{R}$ is the rotation matrix and $\mathrm{P}$ is the translation matrix below [6].

$$
R=\left[\begin{array}{ccc}
n_{x} & o_{x} & p_{x} \\
n_{y} & o_{y} & p_{y} \\
n_{z} & o_{z} & p_{z}
\end{array}\right] \quad P=\left[\begin{array}{c}
p_{x} \\
p_{y} \\
p_{z}
\end{array}\right]
$$

According to D-H matrix method and the mechanical data of KUKA KR5-arc robot, the D-H parameters of manipulator links can be obtained, shown in Table 1, and the results make solid preparation for the kinematics analysis of the robot.

$\mathrm{a}_{i}$ (Link Length) is length between $O_{i}^{\prime}$ and $O_{\mathrm{i}+1}$. This is measured as the distance between the 
common normal to the axes $Z_{\mathrm{i}}$ and $Z_{i+1}$ along $X_{i+1} \cdot \alpha_{\mathrm{i}}$ (Twist Angle) is angle between the orthogonal projections of the joint axes $Z_{\mathrm{i}}$ and $Z_{i+1}$ onto a plane normal to the common normal.

$\mathrm{d}_{i}$ (Joint Offset) is length of the intersections of the common normal on the joint axis $Z_{\mathrm{i}}$, i.e. $O_{i}$ and $O_{i}^{\prime}$. It is the relative position of links $i-1$ and $i$. $\theta_{i}$ (Joint Angle)is angle between the orthogonal projections of the common normal $X_{i}$ and $X_{i+1}$, to a plane normal to the joint axes $Z_{i}$ [7].

Table 1 D-H parameters of manipulator links

\begin{tabular}{cccccl}
\hline$n$ & $\mathrm{a}_{\mathrm{i}} / m m$ & $\alpha_{\mathrm{i}} /\left(^{\circ}\right)$ & $d_{i} / m m$ & $\theta_{\mathrm{i}} /\left(^{\circ}\right)$ & $\theta_{\mathrm{n}} /\left(^{\circ}\right)$ \\
\hline 1 & 180 & 90 & 400 & 0 & $+/-155^{\circ}$ \\
2 & 600 & 0 & 0 & 90 & $+65^{\circ}$ to $-180^{\circ}$ \\
3 & 120 & 90 & 0 & 0 & $+158^{\circ}$ to $-15^{\circ}$ \\
4 & 0 & 90 & 620 & 0 & $+/-350^{\circ}$ \\
5 & 0 & 90 & 0 & 90 & $+/-130^{\circ}$ \\
6 & 0 & 0 & 115 & 0 & $+/-350^{\circ}$ \\
\hline
\end{tabular}

\section{Robotic Toolbox in Simulation \& Kinematics}

Robotic Toolbox can complete the simulation with robot modeling, 3D motion and kinematic parameters. After getting the link matrix $L$ and modeling the robot $R$, the joint angle of initial position $q A$ and final position $q A B$ are determined. The movement time $T$ and the each step time $t_{0}$ are also necessary for the calculation [8].

In this simulation, $\mathrm{L}=\operatorname{link}\left(\left[\begin{array}{lllll}\mathrm{a}_{i} & \alpha_{i} & d_{i} & \theta_{i} & 0\end{array}\right]\right) ; \mathrm{R}=\operatorname{robot}(\{\mathrm{L} 1 \mathrm{~L} 2 \mathrm{~L} 3 \mathrm{~L} 4 \mathrm{~L} 5 \mathrm{~L} 6\}) ; \mathrm{T}=4.5 ; \mathrm{t}_{0}=0.01$; $\mathrm{qA}=\left[\begin{array}{llllll}0 & 0 & 0 & 0 & 0 & 0\end{array}\right] ; \mathrm{qAB}=\left[\mathrm{pi} / 4\right.$ pi/2 pi/8 pi/2 $7^{*} \mathrm{pi} / 6$ pi/2] . Key MATLAB orders are listed below and 3D motion simulation and the trajectory of manipulator see Figure 2 (a) and (b).

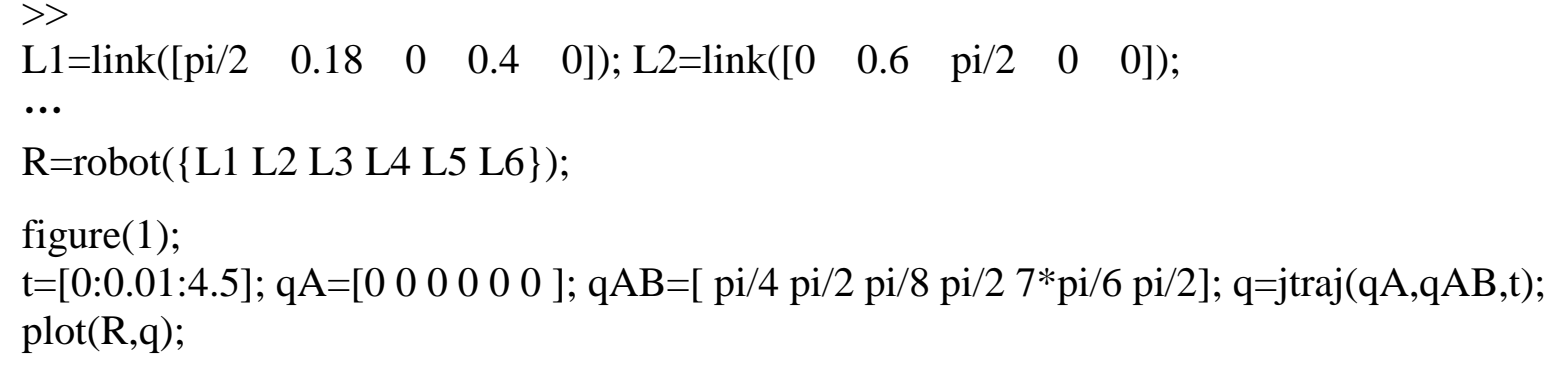

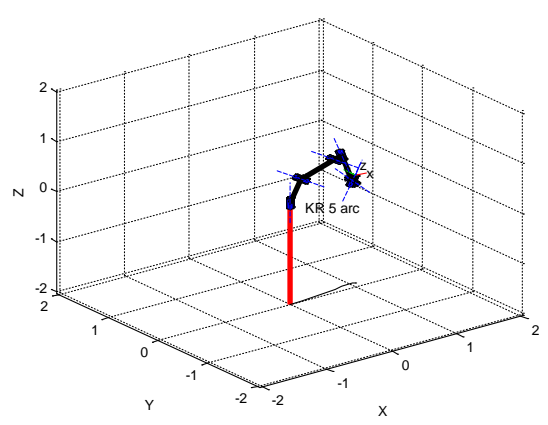

(a) 3D motion

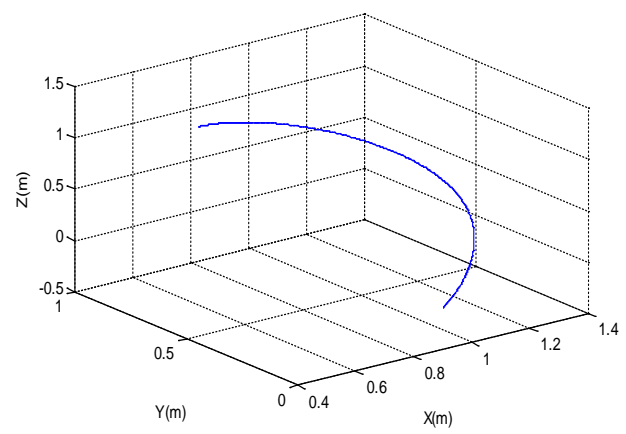

(b) The trajectory

Fig.2. The simulation

In the kinematics analysis, the simulation has been keeping tracking of the position and orientation of the manipulator at each moment [9]. The date recorded can also give us a clear description of the the displacement in three coordinate systems $x / y / z$, in Figure 3 (a) and (b) and (c). 


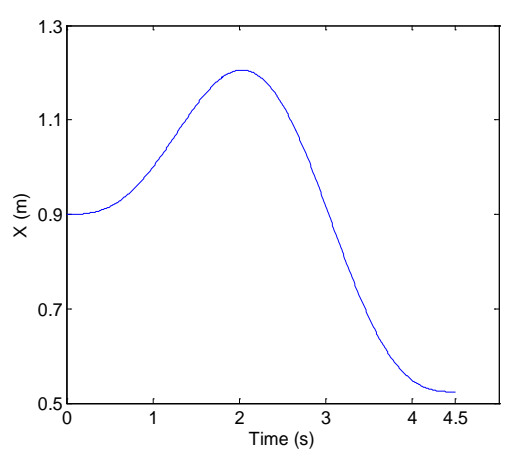

(a) X displacement

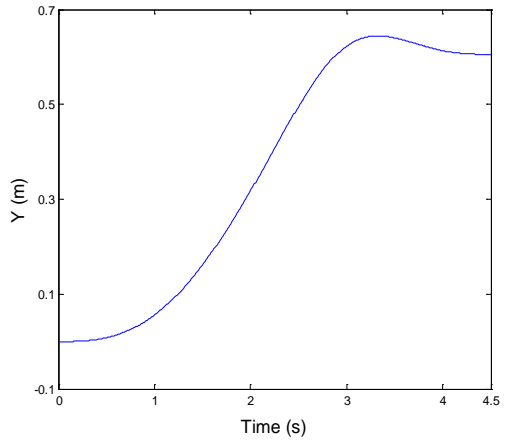

(b) Y displacement

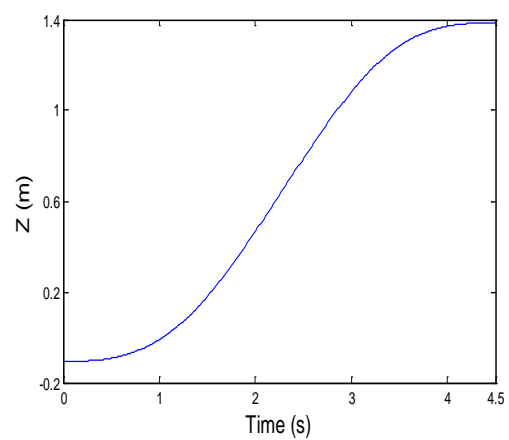

(c) Z displacement

Fig.3. The $\mathrm{X} / \mathrm{Y} / \mathrm{Z}$ displacement

\section{Conclusion}

Based on the Robotic Toolbox in MATLAB, the kinematics and simulation of KUKA KR5-arc welding robot can be analyzed vividly. The simulation draw the working space in 3D motion of the mobile robot. With the results visually on the screen and abundant curve date in the space, we can analysis robot motion accurately and verify the correctness of link parameter designed initially. It is also a foundation of the robot dynamics and control.

\section{References}

[1] XinSong Jiang. Discussion on the development direction of robot technology in the future. Robot. 1996.

[2] ShangYang Lin, Shanben Chen, Chengtong Li. Welding robot and its application. Mechanical Industry Press. 2000.

[3] Lin Wu, GuangJun Zhang, HongMing Gao. Technology of Welding Robot [A]. China Surface Engineering. 2006 (5) 29-35.

[4] S K SAHA. Introduction to Robotics. Mechanical Industry Press. 2015.

[5] HouZhong Wei, Dan Xue, LiQi Jiao, WenFeng Bai. Error Analysis and Simulation based on KUKA six-degree robust [A]. Journal of Changchun University of Technology (Nature Science Edition). 2012 (03) 28-32

[6]Gui CHEN, JianHong WANG, YuDong TANG. Kinematics Simulation and Experimental Research of KUKA robot [A]. Modular Machine Tool \& Automatic Manufacturing Technique. 2014 (08) $94-97$

[7] S K SAHA. Introduction to Robotics. Mechanical Industry Press. 2015.

[8] YongLun Chen, ShiQing Zhu, LiJia Luo, SongGuo Liu. Kinematics Analysis and Simulation of QJ-6R Welding Robot Based on Matlab [A]. Mechanical \& Eleecteical Engineering Magazine. 2007 (11) 07-10

[9] Yu Wang, QiHua Wang, JianGuang Zhao, ZhaoLong Zhang. Research on welding robot trajectory and motion simulation based on virtual prototype technology [A]. Transactions of The China Welding Institution. 2012 (04) 109-112 